\title{
Blue Straggler Stars: A Window Into Alternative Pathway Stellar Products
}

\author{
Natalie M. Gosnell* \\ University of Texas at Austin \\ E-mail: gosnelleastro.as.utexas.edu
}

\begin{abstract}
Our understanding of stellar evolution is built upon a synergy between observations and theory. Careful and thorough observations of open clusters provide a laboratory, of sorts, for testing theories and revealing where our current understanding of stellar evolution falls short. Complete membership studies of open clusters reveal that $25 \%$ of the evolved stars do not agree with simple stellar evolutionary models, but instead follow alternative pathways in stellar evolution. In order to draw a comprehensive picture of stellar evolution we must include these canonically "strange" stars in our definition of standard stellar populations. The majority of these alternative pathway stellar products are blue straggler stars, whose dominant formation mechanism has been an outstanding question for almost six decades. In this talk I will review the current state of blue straggler observations and formation theories, focusing on recent developments in studies of blue straggler stars in cluster environments. I will then present my own work on determining the mass transfer formation frequency of blue straggler stars in open cluster NGC 188 using Hubble ultraviolet photometry, and illustrate how these blue stragglers will help to redefine detailed binary mass transfer models.
\end{abstract}

BASH 2015

18 - 20 October, 2015

The University of Texas at Austin, USA

\footnotetext{
*Speaker.
} 


\section{Introduction}

Our understanding of stellar evolution is built upon a synergy of observations and theory. Open clusters - groups of stars born at the same time and out of the same material — are the closest thing astronomers have to a laboratory for studying stellar evolution. Excellent observations of open cluster populations serve as an important tool for testing theoretical models [1,2]. To this end, it is imperative to have accurate open cluster memberships. Kinematic information from radial velocities [e.g., 3] and proper motions [e.g., 4] identify the co-moving members of a particular cluster out of the bulk motion of stars in the field of the Galaxy. The result of "cleaning" an optical color-magnitude diagram (CMD) in this way is shown in Figure 1 for the massive open clusters M67 and NGC 188.

With cleaned CMDs it is immediately apparent that not all stars in these clusters lie along the traditional expected path of stellar evolution, including the main sequence, subgiant branch, and giant branch of the cluster. Stars with temperatures (observed as colors) and luminosities that fall off of expectations of simple stellar evolution must have followed some alternative evolutionary pathway. These alternative pathway stellar products are classified according to their location in an optical CMD, and are shown on the right-most side of Figure 1. Sub-subgiants (red regions) are stars that lie below the subgiant branch. Yellow giants (yellow regions) are stars hotter or more yellow than the giant branch. Blue straggler stars (BSSs, blue regions) are traditionally defined to be stars bluer and brighter than the main sequence turnoff of the cluster. Given the age of the cluster, stars at the masses of BSSs should have evolved to be red giant branch stars, so they are "straggling" behind their expected evolution.

With cluster membership information we can correctly identify alternative pathway stellar products and complete an accurate census of the stellar population in these clusters. This reveals that for both M67 and NGC 188, approximately 25\% of the evolved stars are classified as alternative pathway stellar products (X-ray binaries and W UMa contact binaries are also included). This means that $25 \%$ of evolved stars are not being accurately described by simple stellar evolutionary models. In order to move toward a more comprehensive picture of stellar evolution we must understand the formation and future evolution of these stars. The majority of alternative pathway stellar products are BSSs, and as such by studying BSSs we can make the largest strides in improving our understanding of stellar evolution and encompassing the full array of stars in our theoretical models of stellar populations.

\section{Blue Straggler formation mechanisms}

BSSs were first discovered about 60 years ago in a photometric study of globular cluster M3 [8], and astronomers have been investigating the formation of BSSs ever since. All formation theories rely on the basic premise that BSSs are formed by adding mass to a main sequence star in some way, but how is the mass added? In cluster environments, there are four main possible scenarios: merger of a contact binary, merger or collision during a dynamical encounter, merger of an inner binary in a hierarchical triple system, and mass transfer from a giant star onto a main sequence companion. (In the rest of this paper, mass transfer will be referred to using the Case A, 

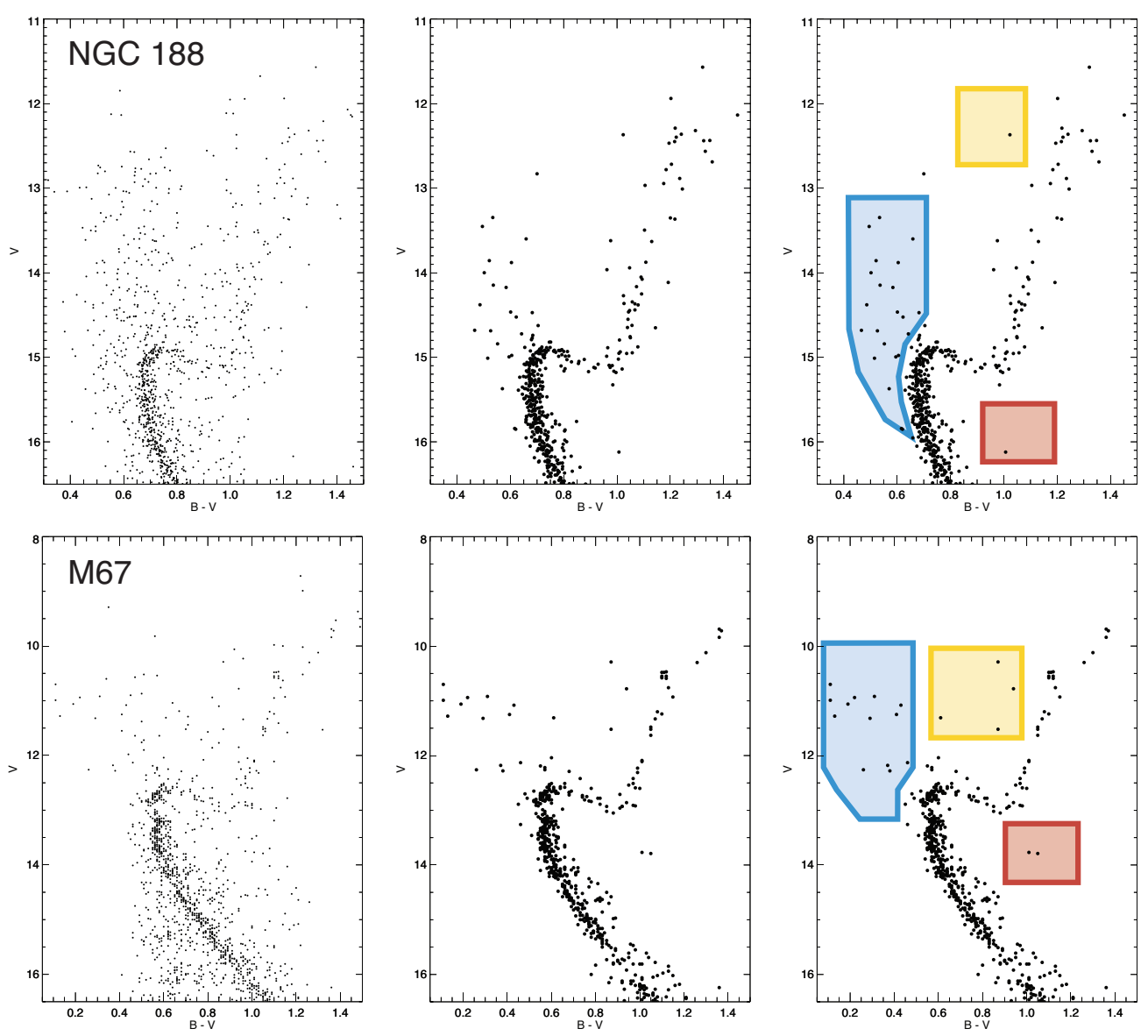

Figure 1: CMDs of NGC 188 (top) and M67 (bottom), showing the impact kinematic membership information has on cleaning CMDs. The left-most figure for each cluster shows all of the 1300-1500 stars in the field of each cluster. Although the main sequence is still visible, detailed studies of the giant branch of each cluster would be difficult. The center figure shows the approximately 500 proper motion and radial velocity cluster members in each cluster. The path of single stellar evolution is very prominent. M67 also exhibits a clean binary sequence above the main sequence. The right-most figure highlights the alternative pathway stellar products: BSSs in blue, yellow giants in yellow, and sub-subgiants in red. These stars are definitely cluster members, but would not have been identified without kinematic membership information. (Adapted from [5], [6], and [7]).

$\mathrm{B}$, and $\mathrm{C}$ nomenclature $[9,10]$. Case $\mathrm{A}$ refers to mass transfer with a main sequence donor, Case $\mathrm{B}$ is with a red giant branch donor, and Case $\mathrm{C}$ is with an asymptotic giant branch donor.)

In order to improve models of stellar evolution it is important to determine which of these formation mechanisms is the most prevalent. In the following sections I briefly summarize some of the recent observations and theory for each formation pathway.

It is important to note that these four very different formation mechanisms can all result in a stellar product that is identified as a "blue straggler". Therefore, the definition of a BSSs is not physical, but empirical. Any star falling above the main sequence turnoff on a cluster CMD will be counted as a BSS, regardless of the mechanisms responsible for creating that object [11]. Therefore, 
progress made in understanding the formation of BSSs may be applicable to stellar products that form through similar mechanisms but do not fall in the BSS region of a CMD.

\subsection{Merger of a contact binary}

After observing the contact binary V1309 Sco coalesce into a single stellar object in real time [12], we now know for certain that it is possible for a contact binary following Case A mass transfer to lead to a single merger product, and those merger products could be observed as BSSs in a cluster environment. However, it is not clear that there are enough Case A progenitor systems to create numerous BSS systems.

Population studies of binaries in the field of the Galaxy show that few are close enough to undergo Case A mass transfer [13]. Of those that do, not all Case A systems become contact binaries [14]. Additionally, theoretical models indicate that few contact binary merger products are massive enough to exceed the main sequence turnoff mass in old open clusters [15]. Although contact binary mergers do occur, and potentially form a few BSSs in a given cluster, it cannot be a dominant formation scenario for BSS populations.

\subsection{Merger driven by Kozai-Lidov mechanism}

Hierarchical triple star systems are stable configurations of a close inner binary orbited by a third star at a much larger distance [16]. It is possible for the third star to induce eccentricity fluctuations in the inner binary, potentially causing the inner binary to merge though a process known as the eccentric Kozai-Lidov mechanism [17, 18, and references therein], as shown in Figure 2. The merger product could be observed as a BSS in a binary with the original tertiary (now secondary) star.

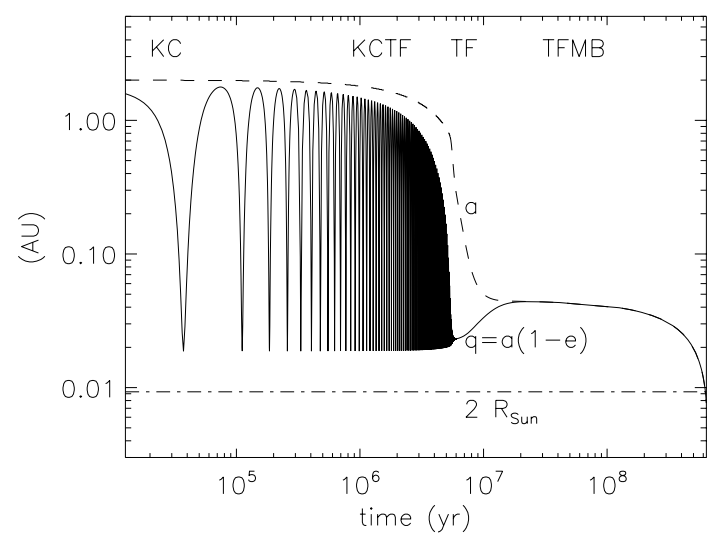

Figure 2: From [17], showing the separation of an inner binary over time with characteristics Kozai-Lidov cycles being driven in a theroretical hierarchical triple system. The inner binary merges after phases of Kozai cycles (KC), tidal friction (TF) and magnetic breaking (MB).

Studies of stellar multiplicity in the field of our Galaxy indicate that the frequency of triple systems range from approximately $20 \%$ for close binaries in the Kepler field [19] to 40\% for systems with inner binary periods less than 10 days [20, 21]. Empirical constraints on the triple frequency in cluster environments are essentially non existent, however, due to the difficulty of detecting triple systems via radial velocity methods. Additionally, triple systems in clusters will be dynamically processed due to encounters with other binaries and triple systems in the cluster environment (see "Dynamical Processing of Stars and Planets Through Star Clusters" by A. M. Geller in these proceedings for a discussion of the impact of dynamics on cluster populations). 
Without strong observational constraints on triple systems in clusters we must rely on theoretical efforts. Current models of cluster populations including the Kozai-Lidov mechanism demonstrate that it unlikely to be a dominant formation pathway for BSSs. Monte Carlo models of globular clusters result in less than $10 \%$ of BSSs forming through Kozai-Lidov cycles [22], and $N$-body models of open clusters yield 2\% Kozai-Lidov BSSs [23]. Future observational measurements of the triple frequency in clusters or identification of specific BSS systems formed through the KozaiLidov mechanism may challenge our current theoretical understanding, but we must turn to other formation pathways to determine the dominant formation mechanisms of cluster BSSs.

\subsection{Collisions during dynamical encounters}

Historically, the study of collisionally-formed BSSs has been analytical, taking into account stellar densities in the core of globular clusters where dynamical encounters are more frequent than in globular cluster halos or in open clusters. With this approach, the number of BSSs in globular cluster cores can be recreated solely through collisions in binary+binary interactions, as shown in Figure 3 [24]. These analytic calculations indicate that collisions in dynamical encounters are the dominant, if not the only, formation mechanism for BSSs in globular cluster cores.

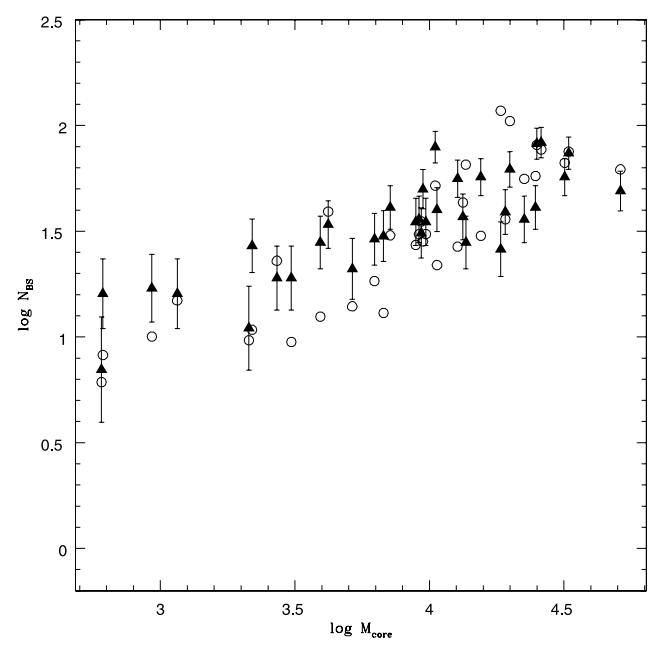

Figure 3: From [24], comparing the number of BSS in the core of globular clusters with the cluster core mass. Observed numbers of BSSs are black triangles and analytically predicted numbers of BSSs are open circles. The analytically calculated number of BSSs match the observations by assuming all the BSSs are created through binary+binary collisions.

Recent improvements in computer power now allow for Monte Carlo models of globular cluster populations. The study of collisionally-formed BSSs in dense environments is no longer constrained to analytical methods. These Monte Carlo models confirm that in dense stellar environments $\left(\rho_{c}>10^{3} \mathrm{M}_{\odot} \mathrm{pc}^{-3}\right)$ collisions in binary+binary interactions dominate the formation of BSSs [25]. Interestingly, observational confirmation of the relationship between the number of BSSs and the encounter rate in globular cluster cores was not found in earlier studies due to poor photometric precision [26, 27], illustrating the importance of quality observations along with detailed thoeretical efforts.

\subsection{Case B or Case C mass transfer}

If collisionally-formed BSSs dominate in high density environments, then another formation mechansim must be responsible for creating most BSSs in lower density environments. Mass 
transfer can create a BSS in a cluster when a main sequence secondary accretes enough mass from a red giant branch or asymptotic giant branch primary star via Roche lobe overflow to exceed the main sequence turnoff mass [e.g., 28]. Mass transfer was one of the first theories put forth for BSS formation [29], but direct observational evidence of BSSs formed through mass transfer has been elusive until recently.

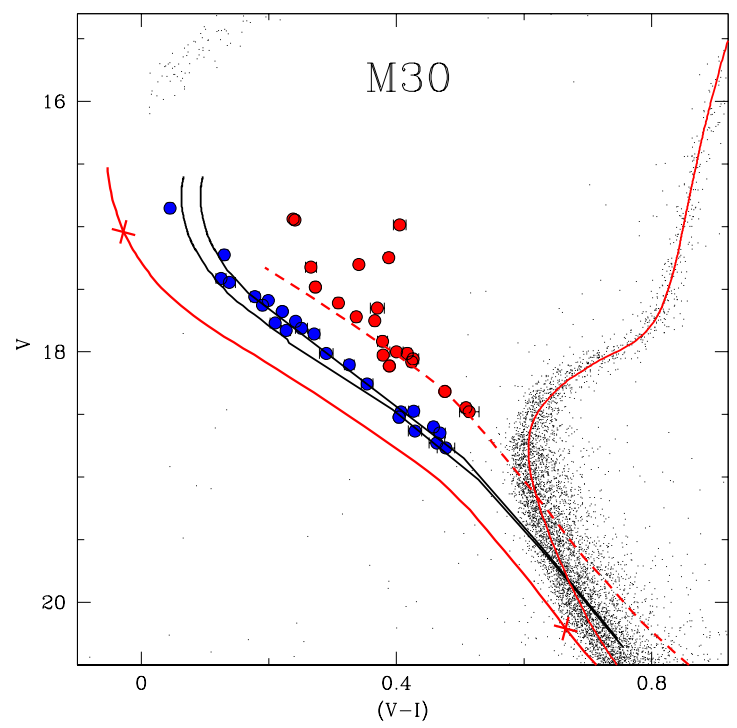

Figure 4: From [30], a CMD of globular cluster M30 showing a double sequence of BSSs, shown as blue and red dots. The solid red lines show the zero age main sequence and the current isochrone for M30. The solid black lines are collisional isochrones that overlap with the collisional sequence of BSSs [31]. The red dashed line marks the low luminosity boundary predicted for mass transfer-formed BSSs.

Observations of globular cluster M30 reveal a remarkable double sequence of BSSs (Figure 4) that the authors claim is from concurrent formation of BSSs via mass transfer and collisions [30]. Indeed, the presence of collisionally-formed BSSs or mass transfer-formed BSSs does not preclude the presence of the other. The bluer sequence of BSSs in M30 aligns well with isochrones for collisionally-formed BSSs [31], while the redder sequence is well-explained with Monte Carlo models of mass transfer products [32]. However, the presence of a double sequence BSS population seems to be unique to M30, so similar techniques cannot be applied in other clusters.

Other work has searched for mass transfer-formed BSSs through abundance measurements. In the mass transfer process, material from the interior of a red giant branch or asymptotic giant branch star is deposited onto the surface of the newly-formed BSS, resulting in abundance anomalies. Six BSSs in globular cluster 47 Tuc have depleted Carbon and Oxygen indicating the BSS progenitor accreted mass from a red giant star with an interior of CNO-processed material [33]. Five BSSs in open cluster NGC 6819 show enhanced Barium (Figure 5), which is an s-process element formed in the interior of asympototic giant branch stars during thermal pulsations [34]. However, what seem to be clear detections of post-mass transfer BSSs have complications. Some of the depleted BSSs in 47 Tuc are currently in contact binaries, which is not consistent with a history of accreting material from an red giant branch star. Four of the five Barium BSSs in NGC 6819 are not in known binaries, which is also inconsistent with a history of accreting material from an asymptotic giant branch star.

In summary, when considering dominant formation mechanisms for BSS in cluster environments we know 1) Case A mergers cannot create enough BSSs to be a dominant formation scenario, 2) mergers in triple systems via the Kozai-Lidov mechanism are not very common, 3) collisions 


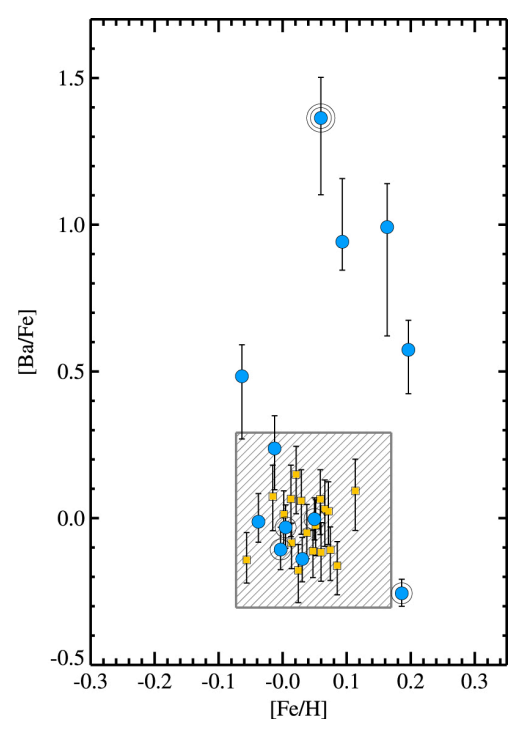

Figure 5: From [34], showing abundance measurements of $[\mathrm{Ba} / \mathrm{Fe}]$ vs. $[\mathrm{Fe} / \mathrm{H}]$ from a study of open cluster NGC 6819 . BSSs are blue circles and main sequence stars are orange squares. BSSs in known binaries are shown with open circles, and a double-lined binary is marked with two circles. Five BSSs show increased Barium, an s-process element formed in the interior of AGB stars during thermal pulses, suggesting that these BSSs formed through mass transfer from an AGB star onto a MS companion. However, the BSSs are either not in detected binaries or in a double-lined binary with a non-AGB star, complicating the proposed mass transfer histories of these systems.

are likely responsible for forming the majority of BSSs in dense stellar environments, and 4) mass transfer must be the dominant formation scenario in lower density environments, but observational evidence for mass transfer formation of BSSs is often conflicting.

\section{HST study of NGC 188 Blue Stragglers}

Many studies of open cluster BSSs in the past 10 years have focused on the 7 Gyr open cluster NGC 188, as it provides an almost ideal environment for studying BSSs. The cluster itself is extremely well-studied, with radial velocity and proper motion membership information $[35,36]$, complete binary information for binaries with periods less than 10,000 days [5], and is the subject of sophisticated $N$-body models [23]. At 7 Gyr, the BSSs in NGC 188 are comparatively cooler than in younger open clusters or in lower metallicity globular clusters, allowing for accurate radial velocity measurements which are necessary to determine membership and binary information.

This work on NGC 188 revealed a population of 21 BSSs (2 short-period binaries, 15 longperiod binaries, and 4 non-velocity variables). The majority of BSSs in NGC 188 are in longperiod binaries [37] and those binary periods are suggestive of BSS formation via Case C mass transfer [38]. I helped lead a Hubble Space Telescope (HST) study designed to convert the previous suggestive results of mass transfer formation into conclusive results.

BSSs formed through Case B or Case C mass transfer will have WD companions, which are the remnant cores of the giant stars that transferred material onto the BSS progenitor. For BSSs in NGC 188, if the mass transfer ended within the past 400 Myr the WD companion will be hot enough to be detected as a far-ultraviolet (FUV) photometric excess above the emission expected for a BSS alone. The presence of any detected WDs can be used to then map out the specific histories of mass transfer-formed BSSs. The following sections outline this work, originally presented in Gosnell et al. (2014) and Gosnell et al. (2015) [7, 39]. 


\subsection{FUV color-magnitude diagram of NGC 188 Blue Stragglers}

The presence of a hot white dwarf companion in a BSS binary changes the FUV emission of the system in a predicatable manner. This is clear in Figure 6. The emission from BSSs without WD companions is faint and red (gray contours), but adding WD companions of increasing temperature results in bright, blue emission (rainbow-colored tracks). These synethetic photometric models are compared to the observed BSSs in NGC 188 (black dots with 2 sigma error bars). Although many BSSs in NGC 188 are consistent with BSS-only FUV emission there are some sources with very obvious FUV excesses.

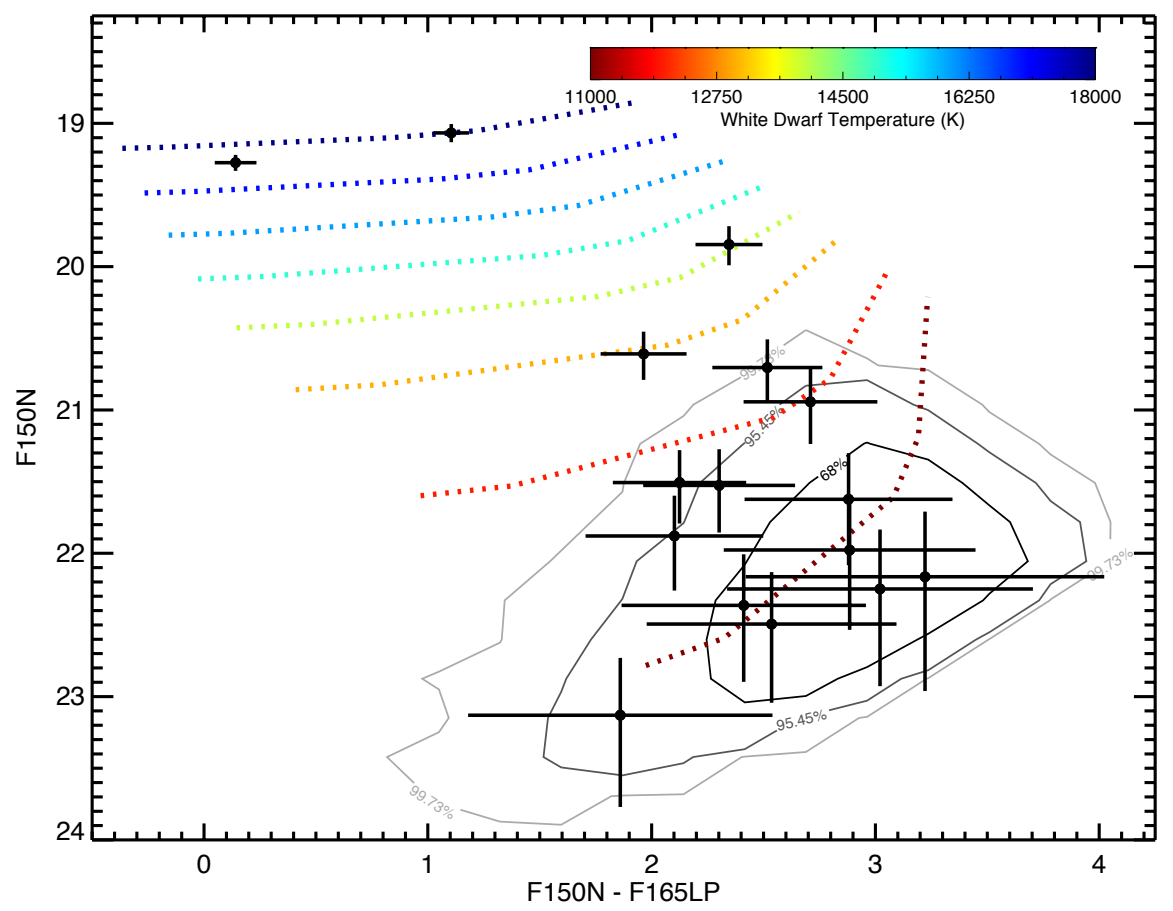

Figure 6: From [39], a FUV CMD of the NGC 188 BSSs. The gray contours show the expected distribution of BSS emission in the FUV. The rainbow-colored tracks show synethic photometry of BSS+WD binaries with increasing WD temperature from 11,000 K to 18,000 K. Hotter WD companions cause the FUV emission to be bluer and brighter, as expected. The black dots with $2 \sigma$ error bars are HST photometry of the NGC 188 BSS population.

\subsubsection{White dwarf detections}

Specific detections of WDs are done on a star-by-star basis, comparing the photometry in each band to synthetic photometry of BSS and BSS+WD binaries. This method reveals four hot $\left(T_{\text {eff }}>12000 \mathrm{~K}\right)$ WD companions and three cool $\left(11000<T_{\text {eff }}<12000 \mathrm{~K}\right)$ WD companions. Individual photometry for the BSS with the four hottest WD companions is shown in Figure 7. These WD detections indicate these seven BSSs formed through recent mass transfer.

The HST photometry also provides an estimate of the WD temperature, which is correlated with the WD age and the time since mass transfer ended. Using WD cooling models, the temperatures detected for these WDs correspond to ages between 70 and $360 \mathrm{Myr}$ [40, 39]. This is 

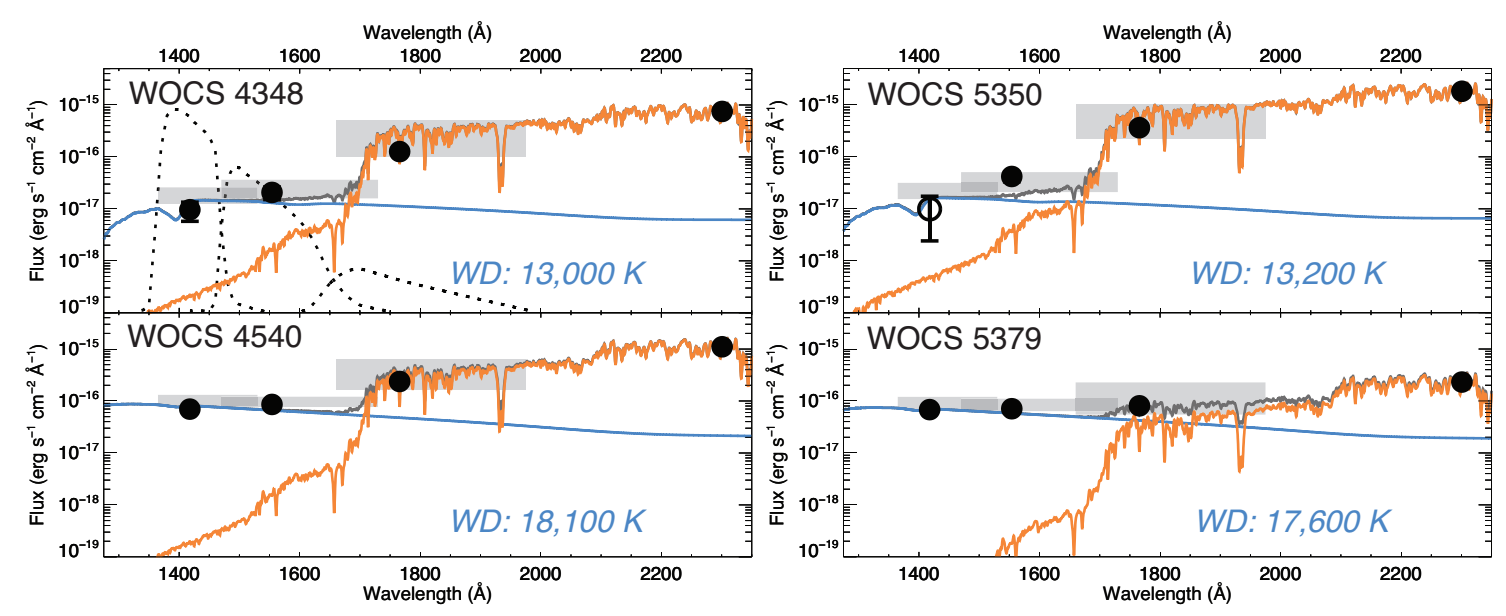

Figure 7: From [39], HST photometry of four BSSs in NGC 188 compared to the best-fit synthetic BSS+WD combination spectra, labeled with WOCS identification numbers. The three bluest photometry points are from HST/ACS/SBC photometry, and the red photometry point is GALEX NUV. The HST bandpasses are shown with black dashed lines in the upper left figure. For these BSSs the FUV emission cannot be explained by a BSS alone, and must be fit by adding a WD at the temperature labeled. The grey boxes show the synthetic photometry of the synthetic BSS+WD pair (shown in orange and blue, respectively), taking into account the uncertainty in the BSS luminosity and temperature.

extremely recent in comparison to the $7 \mathrm{Gyr}$ age of the cluster. This allows us to place unprecedented constraints on the progentior system before mass transfer started.

Since we know how long ago mass transfer ended we can constrain the WD progenitor mass by determining the mass of giant stars in NGC 188 at that time. In order for mass transfer to occur, the radius of that giant star must have been equal to the Roche lobe radius of the progenitor binary, which places constraints on the secondary mass, period, and separation. Finally, the orbit of the binary after mass transfer ends needs to match the observed binary period today [see 7, for some illustrative timelines of BSS formation based on these constraints].

\subsubsection{Mass transfer formation frequency}

The HST FUV photometry reveals seven BSSs with young WD companions, indicating that those seven BSSs formed from recent mass transfer. This photometric method cannot detect WD companions from systems that ended mass transfer more than $400 \mathrm{Myr}$ ago-but those systems are surely there. In order to determine the actual mass transfer formation frequency of BSSs in NGC 188 we must take into account the other possible formation mechansims.

The non-velocity variable BSSs likely formed through collisions in dynamical encounters. Collisions in open cluster environments result in single BSSs or binary BSSs with long enough periods that they would not be detected as binaries through radial velocities [23]. Conversely, mass transfer-formed BSSs should always end up in a detectable binary system. As discussed previously, there are few empirical constraints to inform our assumptions about BSSs formed through the Kozai-Lidov mechanism. Current models suggest that no more than $2 \%$ of binary BSSs in NGC 188 could form this way, resulting in one Kozai-Lidov-formed binary BSS [39]. The two short- 
period BSSs are very interesting systems, but to date their formation has not been successfully modeled, and is potentially the result of stochastic exchanges during dynamical encounters in the cluster environment [23].

This leaves 14 long-period binary BSSs that must have formed through mass transfer, seven of which have detected WD companions. This results in a total mass transfer formation frequency of $67 \%$ for the NGC 188 BSS population.

\subsection{Young BSSs in optical CMD}

Analyzing the location of recently-formed BSSs in an optical CMD (Figure 8) reveals some surprising features. BSS along the zero-age main sequence (ZAMS) are predominantly young, but there are also two young BSSs that are more luminous. Therefore, distance from the ZAMS is not necessarily equivalent with BSS age. Additionally, the two luminous young BSSs have similar luminosities, and therefore masses, as the non-velocity variable BSSs that likely formed through collisions. We do not see any separation in luminosity or color between collisionally-formed and mass transfer-formed BSSs as has been noted in other clusters [30]. In order to determine the formation pathway of a specific BSS one must know the binarity.

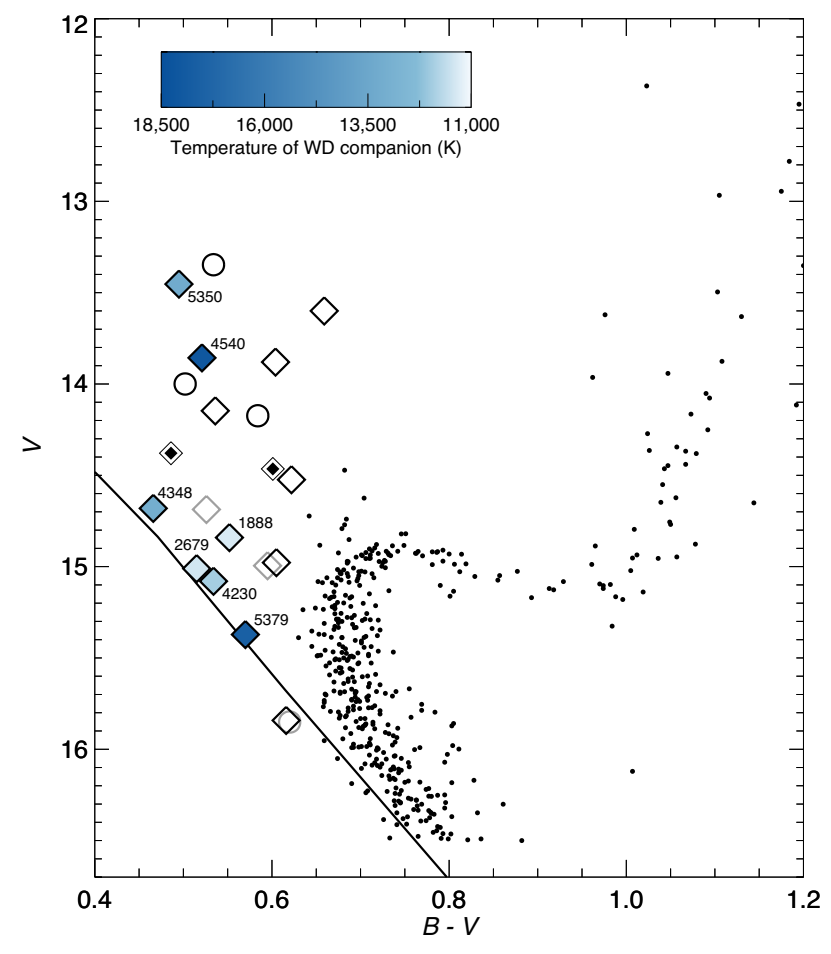

Figure 8: From [39], an optical CMD of NGC 188 highlighting the binarity of the BSSs and the temperature of detected WD companions. Binary BSSs are diamonds and non-velocity variable BSSs are large circles. BSSs with detected WD companions are filled in blue corresponding to the detected WD temperature (given in the colorbar). The solid black line is the zero-age main sequence (ZAMS). It is clear from this figure that most of the BSSs are young due to the WD detections. However, there are two luminous BSSs that are also young. Therefore, distance from the ZAMS is not necessarily correlated with BSS age. Additionally, there is no color or magnitude separation between the BSSs formed through mass transfer and the non-velocity variable BSSs likely formed through collisions.

\section{Summary and Conclusions}

We now know that $25 \%$ of evolved stars in open clusters cannot be described by single star evolution and can be classified as alternative pathway stellar products. These stars, including BSSs, yellow giants, and sub-subgiants, present an exciting new frontier in stellar astrophysics. We can continue to move toward a comprehensive picture of stellar evolution by understanding the formation and future evolution of these stars. BSSs are the majority of alternative pathway stellar 
products, and so by understanding the formation of BSS perhaps we can better understand this subgroup of stars as a whole.

The definition of a BSS is empirical, not physical, so in every evolved stellar population there are multiple BSS formation mechanisms happening concurrently. These include mergers in Case A mass transfer systems, collisions during dynamical encounters, mergers in a hierarchical triple systems via the Kozai-Lidov mechanism, and Case B and C mass transfer. Recent work shows that collisions are the dominant formation mechanism in higher density environments such as the cores of globular clusters, but that Case B or C mass transfer is the dominant formation mechanism in the lower density environments of open clusters.

The direct detection of WD companions to BSSs in NGC 188 presents interesting challenges for $N$-body models. At present, $N$-body models cannot create enough mass transfer-formed BSSs to match observations [23]. Interestingly, these models also create numerous post-common envelope systems that are not observed. If the prescriptions used to send binary systems into common envelope were adjusted so those binaries instead went through stable mass transfer, $N$-body models would exactly match the number and frequency of BSSs observed in NGC 188 [23, 39]. This is further proof that progress in stellar evolution studies are made only through a true synergy of observations and theory.

\section{References}

[1] A. Dotter, B. Chaboyer, D. Jevremović, V. Kostov, E. Baron and J. W. Ferguson, The Dartmouth Stellar Evolution Database, The Astrophysical Journal Supplement 178 (Sept., 2008) 89-101, [0 804 .4473].

[2] A. Bressan, P. Marigo, L. Girardi, B. Salasnich, C. Dal Cero, S. Rubele et al., PARSEC: stellar tracks and isochrones with the PAdova and TRieste Stellar Evolution Code, MNRAS 427 (Nov., 2012) 127-145, [1208.4498].

[3] K. E. Milliman, R. D. Mathieu, A. M. Geller, N. M. Gosnell, S. Meibom and I. Platais, WIYN Open Cluster Study. LX. Spectroscopic Binary Orbits in NGC 6819, The Astronomical Journal 148 (Aug., 2014) 38, [1408.0239].

[4] I. Platais, N. M. Gosnell, S. Meibom, V. Kozhurina-Platais, A. Bellini, C. Veillet et al., WIYN Open Cluster Study. LV. Astrometry and Membership in NGC 6819, The Astronomical Journal 146 (Aug., 2013) 43.

[5] A. M. Geller, R. D. Mathieu, H. C. Harris and R. D. McClure, WIYN Open Cluster Study. XXXVI. Spectroscopic Binary Orbits in NGC 188, The Astronomical Journal 137 (Apr., 2009) 3743-3760, [1512.04991].

[6] A. M. Geller, D. W. Latham and R. D. Mathieu, Stellar Radial Velocities in the Old Open Cluster M67 (NGC 2682). I. Memberships, Binaries, and Kinematics, The Astronomical Journal 150 (Sept., 2015) 97, [1507. 0194 9].

[7] N. M. Gosnell, R. D. Mathieu, A. M. Geller, A. Sills, N. Leigh and C. Knigge, Detection of White Dwarf Companions to Blue Stragglers in the Open Cluster NGC 188: Direct Evidence 
for Recent Mass Transfer, The Astrophysical Journal Letters 783 (Mar., 2014) L8, [1401.7670].

[8] A. R. Sandage, The color-magnitude diagram for the globular cluster M 3., The Astronomical Journal 58 (1953) 61-75.

[9] R. Kippenhahn and A. Weigert, Entwicklung in engen Doppelsternsystemen I. Massenaustausch vor und nach Beendigung des zentralen Wasserstoff-Brennens, Zeitschrift für Astrophysik 65 (1967) 251.

[10] D. Lauterborn, Evolution with mass exchange of case $C$ for a binary system of total mass 7 M sun., Astronomy \& Astrophysics 7 (July, 1970) 150-159.

[11] N. Leigh, A. Sills and C. Knigge, Dissecting the colour-magnitude diagram: a homogeneous catalogue of stellar populations in globular clusters, MNRAS 415 (Aug., 2011) 3771-3782, [1105.3192].

[12] R. Tylenda, M. Hajduk, T. Kamiński, A. Udalski, I. Soszyński, M. K. Szymański et al., V1309 Scorpii: merger of a contact binary, Astronomy \& Astrophysics 528 (Apr., 2011) A114, [1012.0163].

[13] D. Raghavan, H. A. McAlister, T. J. Henry, D. W. Latham, G. W. Marcy, B. D. Mason et al., A Survey of Stellar Families: Multiplicity of Solar-type Stars, The Astrophysical Journal Supplement 190 (Sept., 2010) 1-42, [1007.0414].

[14] B. Tian, L. Deng, Z. Han and X. B. Zhang, The blue stragglers formed via mass transfer in old open clusters, Astronomy \& Astrophysics 455 (Aug., 2006) 247-254, [astro-ph/0604290].

[15] J. C. Lombardi, Jr., J. S. Warren, F. A. Rasio, A. Sills and A. R. Warren, Stellar Collisions and the Interior Structure of Blue Stragglers, The Astrophysical Journal 568 (Apr., 2002) 939-953, [astro-ph/0107388].

[16] E. B. Ford, B. Kozinsky and F. A. Rasio, Secular Evolution of Hierarchical Triple Star Systems, The Astrophysical Journal 535 (May, 2000) 385-401.

[17] H. B. Perets and D. C. Fabrycky, On the Triple Origin of Blue Stragglers, The Astrophysical Journal 697 (June, 2009) 1048-1056, [0901.4328].

[18] S. Naoz and D. C. Fabrycky, Mergers and Obliquities in Stellar Triples, The Astrophysical Journal 793 (Oct., 2014) 137, [1 405 . 5223].

[19] S. Rappaport, K. Deck, A. Levine, T. Borkovits, J. Carter, I. El Mellah et al., Triple-star Candidates among the Kepler Binaries, The Astrophysical Journal 768 (May, 2013) 33, [1302.0563].

[20] A. A. Tokovinin, On the multiplicity of spectroscopic binary stars, Astronomy Letters 23 (Nov., 1997) 727-730. 
[21] T. Pribulla and S. M. Rucinski, Contact Binaries with Additional Components. I. The Extant Data, The Astronomical Journal 131 (June, 2006) 2986-3007, [astro-ph / 0601610 ].

[22] F. Antonini, S. Chatterjee, C. L. Rodriguez, M. Morscher, B. Pattabiraman, V. Kalogera et al., Black Hole Mergers and Blue Stragglers from Hierarchical Triples Formed in Globular Clusters, The Astrophysical Journal 816 (Jan., 2016) 65, [1509.05080].

[23] A. M. Geller, J. R. Hurley and R. D. Mathieu, Direct N-body Modeling of the Old Open Cluster NGC 188: A Detailed Comparison of Theoretical and Observed Binary Star and Blue Straggler Populations, The Astronomical Journal 145 (Jan., 2013) 8, [1210.1575].

[24] N. Leigh, A. Sills and C. Knigge, An analytic model for blue straggler formation in globular clusters, MNRAS 416 (Sept., 2011) 1410-1418, [1105. 5388].

[25] S. Chatterjee, F. A. Rasio, A. Sills and E. Glebbeek, Stellar Collisions and Blue Straggler Stars in Dense Globular Clusters, The Astrophysical Journal 777 (Nov., 2013) 106, [1302.7284].

[26] M. B. Davies, G. Piotto and F. de Angeli, Blue straggler production in globular clusters, MNRAS 349 (Mar., 2004) 129-134, [astro-ph/ 0401502 ].

[27] N. Leigh, A. Sills and C. Knigge, Where the Blue Stragglers Roam: Searching for a Link between Formation and Environment, The Astrophysical Journal 661 (May, 2007) 210-221, [astro-ph/0702349].

[28] X. Chen and Z. Han, Mass transfer from a giant star to a main-sequence companion and its contribution to long-orbital-period blue stragglers, MNRAS 387 (July, 2008) 1416-1430, [0804.2294].

[29] W. H. McCrea, Extended main-sequence of some stellar clusters, MNRAS 128 (1964) 147.

[30] F. R. Ferraro, G. Beccari, E. Dalessandro, B. Lanzoni, A. Sills, R. T. Rood et al., Two distinct sequences of blue straggler stars in the globular cluster M 30, Nature 462 (Dec., 2009) 1028-1031, [1001.1096].

[31] A. Sills, A. Karakas and J. Lattanzio, Blue Stragglers After the Main Sequence, The Astrophysical Journal 692 (Feb., 2009) 1411-1420, [0811.2974].

[32] Y. Xin, F. R. Ferraro, P. Lu, L. Deng, B. Lanzoni, E. Dalessandro et al., The Binary Mass Transfer Origin of the Red Blue Straggler Sequence in M30, The Astrophysical Journal 801 (Mar., 2015) 67, [1501.01358].

[33] F. R. Ferraro, E. Sabbi, R. Gratton, G. Piotto, B. Lanzoni, E. Carretta et al., Discovery of Carbon/Oxygen-depleted Blue Straggler Stars in 47 Tucanae: The Chemical Signature of a Mass Transfer Formation Process, The Astrophysical Journal Letters 647 (Aug., 2006) L53-L56, [astro-ph/0610081]. 
[34] K. E. Milliman, R. D. Mathieu and S. C. Schuler, Barium Surface Abundances of Blue Stragglers in the Open Cluster NGC 6819, The Astronomical Journal 150 (Sept., 2015) 84, [1508.01236].

[35] I. Platais, V. Kozhurina-Platais, R. D. Mathieu, T. M. Girard and W. F. van Altena, WIYN Open Cluster Study. XVII. Astrometry and Membership to V=21 in NGC 188, The Astronomical Journal 126 (Dec., 2003) 2922-2935, [astro-ph/0309749].

[36] A. M. Geller, R. D. Mathieu, H. C. Harris and R. D. McClure, WIYN Open Cluster Study. XXXII. Stellar Radial Velocities in the Old Open Cluster NGC 188, The Astronomical Journal 135 (June, 2008) 2264-2278, [1512.04983].

[37] R. D. Mathieu and A. M. Geller, A binary star fraction of 76 per cent and unusual orbit parameters for the blue stragglers of NGC 188, Nature 462 (Dec., 2009) 1032-1035.

[38] A. M. Geller and R. D. Mathieu, A mass transfer origin for blue stragglers in NGC 188 as revealed by half-solar-mass companions, Nature 478 (Oct., 2011) 356-359, [1110 . 3793].

[39] N. M. Gosnell, R. D. Mathieu, A. M. Geller, A. Sills, N. Leigh and C. Knigge, Implications for the Formation of Blue Straggler Stars from HST Ultraviolet Observations of NGC 188, The Astrophysical Journal 814 (Dec., 2015) 163, [1510.04290].

[40] J. B. Holberg and P. Bergeron, Calibration of Synthetic Photometry Using DA White Dwarfs, The Astronomical Journal 132 (Sept., 2006) 1221-1233. 